IASSNS-HEP-99-93

KCL-MTH-99-44

LAPTH-754/99

July 5,2021

\title{
Extremal correlators in four-dimensional SCFT
}

\author{
B. Eden ${ }^{a, b}$, P.S. Howe ${ }^{a}$, C. Schubert ${ }^{b, c}$, E. Sokatchev ${ }^{b}$ and P.C. West ${ }^{a}$ \\ a Department of Mathematics, King's College, London, UK \\ ${ }^{b}$ Laboratoire d'Annecy-le-Vieux de Physique Théoriqu円 LAPTH, Chemin de Bellevue, B.P. 110, \\ F-74941 Annecy-le-Vieux, France \\ ${ }^{c}$ School of Natural Sciences, Institute for Advanced Study, Olden Lane, Princeton, NJ 08540, US
}

\begin{abstract}
It is shown that certain extremal correlators in four-dimensional $N=2$ superconformal field theories (including $N=4$ super-Yang-Mills as a special case) have a free-field functional form. It is further argued that the coupling constant dependence receives no correction beyond the lowest order. These results hold for any finite value of $N_{c}$.
\end{abstract}

\footnotetext{
${ }^{1}$ UMR 5108 associée à l'Université de Savoie
} 


\section{Introduction}

In an interesting recent article D'Hoker et al [1] have shown that certain "extremal" correlation functions are free in AdS supergravity. These authors also gave some arguments for this to be true for the CFT correlators corresponding to those supergravity correlators according to the Maldacena conjecture [2]. Further one-loop and instanton checks have been given in [3]. In this note we show that the non-renormalisation of such correlators can be understood very simply in non-perturbative superconformal field theory as a consequence of the superconformal Ward identities and the constrained nature of the harmonic superfields that appear in the theory. We further show that these simplifications hold in any finite $N=2$ SCFT and not just the $N=4$ SYM theory (at least for the correlators of $N=2$ matter multiplets). We also argue that the result could be extended to another class of "subextremal" correlators.

The reason that the Ward identities in superconformal theories are stronger than one might naively expect is not because some of the invariants, i.e. cross-ratios, that occur in non-supersymmetric conformal field theories do not have supersymmetric extensions, but rather because all the basic field strengths and matter multiplets in four-dimensional supersymmetric theories are described by constrained superfields. These constraints, when taken together with the superconformal Ward identities, may lead to much stronger results than those that follow for non-supersymmetric theories from conformal invariance alone. For example, the chiral nature of the matter superfield in $N=1$ theories can be exploited to determine the anomalous dimension of operators composed from this field 何. The same is true for the gauge field strength superfields in $N=1$ and $N=2$ Yang-Mills. The remaining supermultiplets that occur in four-dimensional theories with rigid extended supersymmetry are the $N=2$ matter multiplet and the $N=4$ Yang-Mills multiplet. The former has an off-shell harmonic superspace formulation [5] which can be used to give a complete description of the quantum theory including its Feynman rules and superconformal properties. While only an on-shell harmonic superspace formulation [6] is known for the latter it can still be used to investigate the properties of the correlation functions under superconformal transformations. Both of these multiplets are described by superfields which satisfy simple constraints: the superfields obey the condition of Grassmann (G-)analyticity (an analog of chirality in $N=1$ theories) and are harmonic (H-)analytic (a dynamical property of $N=2$ matter [5] which can also be extended to $N=4$ SYM [6]). These constraints mean that such superfields depend on only half of the number of Grassmann odd coordinates in the full superspace, as in the case of chiral superfields, and that they depend analytically on the coordinates of the internal coset space which is adjoined to super Minkowski space to form harmonic superspace. We shall refer to such superfields as analytic superfields in the following.

In references [7, 8] a systematic study of the superconformal Ward identities and their consequences for Greens functions of $N=2$ and $N=4$ analytic operators was initiated. The $N=2$ operators are gauge-invariant products of the hypermultiplet $q$ and the $N=4$ operators are gauge-invariant products of the $N=4$ field strength $W$. (We shall use the notation $\mathcal{O}_{p}$ to denote operators of the form $\operatorname{tr}\left(q^{p}\right)$ or $\operatorname{tr}\left(W^{p}\right)$.) This approach has led to a number of interesting results [7, 8], in particular: the $N=4 \mathrm{SYM}$ field strength is a covariantly analytic scalar superfield $W$ from which the aforementioned set of analytic gauge-invariant operators can be built; the two- and three-point Greens functions of these operators were determined up to constants [7, 9] (see also [10]); the set of all non-nilpotent analytic superconformal invariants was given [8]. It was also conjectured that the superconformal Ward identities and the constrained nature of the harmonic superfields might be sufficiently strong to determine certain correlation functions up to constants [7, 8]. 
It has subsequently emerged that the $N=4$ operators considered above play an important rôle in the Maldacena conjecture and are in one-to-one correspondence with the Kaluza-Klein multiplets of IIB supergravity on $A d S_{5} \times S^{5}$ [11]. However, although the Ward identities, when combined with Grassmann and harmonic analyticity, do put constraints on the functions of superinvariants that can arise for correlators with four or more points, these constraints do not seem to be enough [12, 13 to determine completely the $N=2$ correlators that contain four harmonic composite matter fields of charge two, contrary to the claim for future work made in [7, [- The situation is very different, however, for four-point correlation functions of analytic operators for which the sum of three of the charges (i.e. powers of $W$ or $q$ in a given operator) is equal to the charge on the fourth. These are the extremal correlators discussed in [1, 30.

In this paper we consider such extremal correlation functions in $N=4$ or $N=2$ SYM theories. They have the form

$$
<\mathcal{O}_{p_{1}} \mathcal{O}_{p_{2}} \mathcal{O}_{p_{3}} \mathcal{O}_{p_{4}}>
$$

where $p_{4}=\sum_{i=1}^{3} p_{i}$. We shall show that the superconformal Ward identities together with analyticity imply that they can be solved for and that they can be expressed as products of two-point functions. This will demonstrate part of the conjecture of D'Hoker et al [1], but it is a stronger result in the sense that it is valid for any gauge group $S U\left(N_{c}\right)$ and not only in the limit $N_{c} \rightarrow \infty$. Furthermore, these results hold also for any $N=2$ SCFT and not just $N=4$, at least for composite operators made out of hypermultiplets. The reason why these correlators are soluble, while the case $p_{1}=\ldots=p_{4}=2$ considered in [12, 13] is not, is related to their different forms when expressed in terms of products of two-point functions multiplied by a function of superconformal invariants. The singularities of the latter in the internal coordinates must be compensated for by the zeros of the former, because all correlation functions have to be analytic. For the extremal correlators the structure of the two-point function prefactor allows essentially no freedom for the function of invariants; it must be a constant.

The superconformal Ward identities alone do not determine the dependence of a correlator on the coupling constant. However, information on this dependence can be extracted by using the reduction formula, first introduced in the context of four-dimensional SCFT by Intriligator [14]. This formula relates the derivative of an $n$-point function with respect to the coupling to an $(n+1)$-point function with one insertion of a bilinear operator and an integration over the insertion point. In the $N=4$ case the inserted operator is the supercurrent $T=\mathcal{O}_{2}$ while in the $N=2$ case the insertion into correlators of G-analytic superfields is the chiral pure SYM Lagrangian. Consideration of this reduction formula led to the realisation [14] that there must be nilpotent superinvariants since only such invariants can contribute in the integration over the insertion point. Nilpotent invariants exist for correlators with five or more points [15, 16]. Their absence for $(n \leq 4)$-point correlators has been used to argue that two-and three-point correlators of analytic operators are not renormalised [15]. Here we shall use the reduction formula to argue that the extremal four-point correlators are not renormalised as a consequence of the absence of nilpotent invariants with the required analyticity properties. We also show that the same argument implies the non-renormalisation of another class of operators, "subextremal" for which $p_{4}=\sum_{i=1}^{3} p_{i}-2$. Note, however, that when using the reduction formula we make the (plausible) assumption that there are no undesired contributions from contact terms [16, 17]. 


\section{Extremal correlators as constrained superconformal covariants}

Let us begin by considering an $n$-point correlator in $N=2$ or $N=4$ SCFT for the operators $\mathcal{O}_{p}$ discussed above. Such a correlator, if its leading term in an expansion in odd coordinates does not vanish, can be written in the form

$$
\mathcal{G}:=<\mathcal{O}_{p_{1}} \mathcal{O}_{p_{2}} \ldots \mathcal{O}_{p_{n}}>=\prod_{r<s}\left(g_{r s}\right)^{m_{r s}} F
$$

where $F$ is some function of the invariants, the $m_{r s} \equiv m_{s r}$ are non-negative integers and the $g_{r s}$ are the free two-point functions of charge one operators (in $N=2$ these are just the hypermultiplet propagators). It is easy to see that this form for $\mathcal{G}$ does solve the superconformal Ward identities because the propagator satisfies

$$
\left(V_{r}+V_{s}+\Delta_{r}+\Delta_{s}\right) g_{r s}=0
$$

where $V$ is the vector field generating a superconformal transformation in analytic superspace and $\Delta$ is the associated analytic density function, and because the transformation rule for a field of charge $p$ is

$$
\delta \mathcal{O}_{p}=V \mathcal{O}_{p}+p \Delta \mathcal{O}_{p}
$$

The $m_{r s}$ must obey the equations

$$
p_{r}=\sum_{s, r \neq s} m_{r s}
$$

whence it follows that

$$
-p_{n}+\sum_{r=1}^{n-1} p_{r}=\sum_{r, s=1}^{n-1} m_{r s} .
$$

In the extremal case $p_{n}=\sum_{r=1}^{n-1} p_{r}$ the solution to this equation is unique. Thus we have

$$
<\mathcal{O}_{p_{1}} \mathcal{O}_{p_{2}} \ldots \mathcal{O}_{p_{n}}>=\prod_{r=1}^{p-1}\left(g_{r n}\right)^{p_{r}} F \quad \text { for } p_{n}=\sum_{r=1}^{n-1} p_{r} .
$$

To be more concrete we now focus on four-point extremal correlators in the $N=2$ theory. The simplest example is $\mathcal{G}:=<\mathcal{O}_{2}(1) \mathcal{O}_{2}(2) \mathcal{O}_{2}(3) \mathcal{O}_{6}(4)>$ where the $\mathcal{O}$ 's are made out of hypermultiplets. It can be written in the following form:

$$
\mathcal{G}=\left(g_{14}\right)^{2}\left(g_{24}\right)^{2}\left(g_{34}\right)^{2} F
$$

where $F$ is a function of super-invariants. The two-point function $g_{r s}$ is the propagator for free hypermultiplets,

$$
g_{r s}=\frac{\hat{y}_{r s}}{x_{r s}^{2}}
$$

where $x_{r s}=x_{r}-x_{s}$, etc., and $\hat{y}_{r s}=y_{r s}-\pi_{r s} x_{r s}^{-1} \lambda_{r s}$. The variables $\lambda$ and $\pi$ are the fermionic coordinates of analytic superspace carrying undotted and dotted spinor indices respectively and $y$ is the standard local coordinate on the coset space $U(1) \backslash S U(2)=\mathbb{C P}^{1}$.

Let us now discuss the function $F$ depending on the superconformal invariants. For four points there are no nilpotent invariants [15], which means that the supersymmetric extension of an 
ordinary Minkowski or internal space invariant is unique, or in other words that all invariants are specified by their body. The two non-trivial invariants with respect to the $x$ conformal transformations are the cross-ratios

$$
s=\frac{x_{14}^{2} x_{23}^{2}}{x_{12}^{2} x_{34}^{2}} \quad t=\frac{x_{13}^{2} x_{24}^{2}}{x_{12}^{2} x_{34}^{2}}
$$

and the non-trivial invariant with respect to the $y$ conformal transformations is

$$
v=\frac{y_{14} y_{23}}{y_{12} y_{34}} .
$$

The quantity $w=\frac{y_{13} y_{24}}{y_{12} y_{34}}$ is conformally invariant, but can be written in terms of $v$, namely $w=1+v$. Thus for four points in analytic superspace, there are three independent invariants. Any basis set whose body can be solved for the cross-ratios $s, t, v$ may be chosen, for instance [8]

$$
S=\frac{s \operatorname{det} X_{14} s \operatorname{det} X_{23}}{s \operatorname{det} X_{12} s \operatorname{det} X_{34}}, T=\frac{s \operatorname{det} X_{13} s \operatorname{det} X_{24}}{s \operatorname{det} X_{12} s \operatorname{det} X_{34}}
$$

and

$$
U=\operatorname{str}\left(\mathrm{X}_{34}^{-1} \mathrm{X}_{41} \mathrm{X}_{12}^{-1} \mathrm{X}_{23}\right)
$$

where $X$ is a $(2 \mid N)$ by $(2 \mid N)$ matrix coordinatising a patch on analytic superspace and $X_{r s}$ denotes a coordinate difference.

The superconformal invariants $S, T$ can be written as

$$
S=\frac{s}{\hat{v}}, T=\frac{t}{\hat{w}}
$$

where

$$
\hat{v}=\frac{\hat{y}_{23} \hat{y}_{14}}{\hat{y}_{12} \hat{y}_{34}}, \quad \hat{w}=\frac{\hat{y}_{13} \hat{y}_{24}}{\hat{y}_{12} \hat{y}_{34}} .
$$

It is clear that both of these objects and also $U$ contain singularities in $y$. At the same time, a correlation function of gauge-invariant composite operators made out of hypermultiplets must be analytic in $y$. This implies that the function $F$ in equation (6) must depend on the superinvariants in such a way that the singularities in $y$ that appear in $F$ are cancelled by the zeros in $y$ contained in the propagators. Furthermore, this must be true to all orders in $\lambda \pi$. To examine this issue we will need to know the dependence of the superinvariants on $y$ and $\lambda, \pi$. It turns out that the singularities cannot be cancelled in the extremal case, so that the function $F$ can only be a constant.

We write the nilpotent parts of $\hat{w}$ and the superconformal invariant $U$ as $\Delta w$ and $\Delta U$ respectively, namely

$$
\hat{w}=1+\hat{v}+\delta w, \quad U=1-t+s+\hat{v}+\Delta U
$$

According to the argument above we can, by taking appropriate functions of $S, T, U$, construct three superconformal invariants whose leading terms are given by $s, t$ and $\frac{v}{s}$. These superconformal invariants are

$$
S^{\prime}=S V, \quad T^{\prime}=T(1+V), \quad z=\frac{1}{S}
$$


where $V=\frac{T+U-1}{1+S-T}$. To first order in $\Delta w$ and $\Delta U$ we find

$$
\begin{aligned}
S^{\prime} & =s+\Delta_{s}=s-\frac{t}{R} \frac{\Delta w}{(1+s z)}+\frac{\Delta U}{R}+\ldots \\
T^{\prime} & =t+\Delta_{t}=t+(1+z)-\frac{t}{R} \frac{\Delta w}{(1+s z)}+\frac{z t}{(1+s z)} \frac{\Delta U}{R}+\ldots \\
z & =\frac{\hat{v}}{s}
\end{aligned}
$$

with $R=(1+z)-\frac{z t}{1+s z}$.

Finally, we are in a position to show that $F$ is a constant as a consequence of the analyticity of the extremal correlators in their dependence on $y$. Taylor-expanding to first order in $\Delta U$ and $\Delta w$ we find that

$$
F\left(s+\Delta_{s}, t+\Delta_{t}, z\right)=F(s, t, z)+\frac{\Delta U}{R} D F-\frac{t \Delta w}{R(1+s z)} \hat{D} F+\ldots
$$

where

$$
D:=\frac{\partial}{\partial s}+\frac{z t}{1+s z} \frac{\partial}{\partial t}, \quad \hat{D}:=\frac{\partial}{\partial s}+(1+z) \frac{\partial}{\partial t} .
$$

Now, the correlation function must be analytic, but the variable $z$ contains a singularity in $y_{12} y_{34}$. Strictly speaking, we consider the singularities in $\hat{y}_{12} \hat{y}_{34}$. It is a special feature of the extremal case that the factors of $\hat{y}$ in the two-point functions that multiply $F$ can never cancel such a singularity and hence $F$ does not depend on $z$. We now consider the terms in $F$ which are first order in $\lambda \pi$, that is, in $\Delta U$ and $\Delta w$. Since these terms have independent spinor structures and go like $v$ and we are taking $v \rightarrow 0$, we must conclude that

$$
D F=0
$$

and

$$
\hat{D} F=0
$$

By considering the coefficient of each power of $z$ in the first equation, it is straightforward to show that $F$ is actually a constant. Clearly, the second equation is then automatically satisfied.

The same conclusion can be reached using $N=4$ harmonic superspace for a similar correlator with three charge two operators (i.e. supercurrents) and one charge six operator. In this case one has the same form for $\mathcal{G}$ but the two-point function is now different. It takes the form

$$
g_{r s}=\frac{\hat{y}_{r s}^{2}}{x_{r s}^{2}}
$$

where $\hat{y}_{r s}=y_{r s}-\pi_{r s} x_{r s}^{-1} \lambda_{r s}$ as before, the only difference being that the odd coordinates and $y$ now carry internal indices as well. The coordinates of $N=4$ analytic superspace are $\left(x^{\alpha \dot{\alpha}}, \lambda^{\alpha a^{\prime}}, \pi^{a \dot{\alpha}}, y^{a a^{\prime}}\right)$ where each index can take on two values and the internal indices are treated in the same way as the spacetime spinor indices. In the $N=4$ case there are four independent super-invariants at four points corresponding to the fact that there are two independent spacetime cross-ratios and two independent internal space cross-ratios each of which has a unique extension to a super-invariant. Again, all of these are singular in such a way that the singularities cannot be cancelled by the prefactor $\Pi g^{m}$ in the extremal correlator, and hence we conclude that the only function of super-invariants that one can have is in fact a constant. 


\section{Four-point correlators and the reduction formula}

We now consider the question of non-renormalisation of the coefficient multiplying the propagators in the expression for the four-point correlators discussed above. This can be done either in the $N=4$ formalism or in $N=2$. In this note we shall give the $N=2$ version since it is applicable to an arbitrary $N=2$ superconformal theory. In this sense the results obtained are stronger than the $N=4$ ones, although they only apply to the hypermultiplet sector of the theory. Furthermore, in this section we shall use the manifestly $S U(2)$-covariant harmonic superspace formalism of reference [5].

The four-point correlators we discuss involve gauge-invariant composite operators $\mathcal{O}_{p_{r}}=\operatorname{tr}\left(q^{p_{r}}\right)$ made out of $p_{r}$ hypermultiplets:

$$
\left\langle\mathcal{O}_{p_{1}}(1) \ldots \mathcal{O}_{p_{4}}(4)\right\rangle \equiv\left\langle p_{1} p_{2} p_{3} p_{4}\right\rangle
$$

The requirement of gauge invariance puts the natural restriction $p_{r} \geq 2$ on the allowed values of the charges. As stated earlier, such correlators should have two fundamental properties: superconformal covariance (follows from the finiteness of the theory) and harmonic (H-)analyticity. The latter is a dynamical property which can be explained as follows.

In the $S U(2)$-covariant harmonic superspace the hypermultiplet is described off shell by a Grassmann (G-)analytic superfield $q^{+}\left(x_{A}, \theta^{+}, \bar{\theta}^{+}, u^{ \pm}\right)$. In it the harmonic variables are defined as $S U(2)$ matrices,

$$
u \in S U(2) \quad \Rightarrow \quad u_{i}^{-}=\left(u^{+i}\right)^{*}, \quad u^{+i} u_{i}^{-}=1
$$

where the $S U(2)$ indices $i=1,2$ are raised and lowered in the usual way. All harmonic functions are homogeneous under the action of a $U(1)$ group counting the charges (like \pm for $u^{ \pm}$), so that they effectively live on the coset $S^{2} \sim U(1) \backslash S U(2)$. Further, the Grassmann variables

$$
\theta^{+\alpha}=u_{i}^{+} \theta^{i \alpha}, \quad \bar{\theta}^{+\dot{\alpha}}=u_{i}^{+} \bar{\theta}^{i \dot{\alpha}}
$$

are $S U(2)$-invariant $U(1)$ projections of the full superspace ones $\theta^{i}, \bar{\theta}^{i}$. Finally, $x_{A}^{\alpha \dot{\alpha}}=x^{\alpha \dot{\alpha}}-$ $4 i \theta^{(i \alpha} \bar{\theta}^{j)} \dot{\alpha} u_{i}^{+} u_{j}^{-}$together with $\theta^{+}, \bar{\theta}^{+}$and $u^{ \pm}$form a basis in the G-analytic superspace closed under the full $N=2$ superconformal group.

It should be stressed that the off-shell hypermultiplet superfield $q^{+}\left(x_{A}, \theta^{+}, \bar{\theta}^{+}, u^{ \pm}\right)$involves an infinite number of auxiliary fields coming from its expansion on $S^{2}$. On shell $q^{+}$obeys the free field equation

$$
D^{++} q^{+}\left(x_{A}, \theta^{+}, \bar{\theta}^{+}, u^{ \pm}\right)=0
$$

where

$$
D^{++}=u^{+i} \partial / \partial u^{-i}-2 i \theta^{+\alpha} \bar{\theta}^{+\dot{\alpha}} \partial / \partial x_{A}^{\alpha \dot{\alpha}}
$$

is the harmonic derivative in G-analytic superspace. Expanding equation (28) in both the Grassmann and harmonic variables, one can show that all the auxiliary fields are eliminated and the remaining physical scalars and spinors are put on shell.

The above off-shell formulation of the hypermultiplet allows one to develop standard Feynman rules [18]. In this context one can argue that the correlator (25) obeys equations of the SchwingerDyson type, e.g., at point $r$,

$$
D_{r}^{++}\left\langle p_{1} p_{2} p_{3} p_{4}\right\rangle=\text { contact terms }, \quad r=1, \ldots, 4 .
$$


In our analysis of superconformal covariants we always make the assumption that the points are kept apart in order to avoid space-time singularities. Then eq. (30) becomes the condition of exact H-analyticity:

$$
D_{r}^{++}\left\langle p_{1} p_{2} p_{3} p_{4}\right\rangle=0, \quad r=1, \ldots, 4 \quad \text { if point } 1 \neq \ldots \neq \text { point } 4 .
$$

As we have seen in section 2, the combination of superconformal covariance and H-analyticity puts strong restrictions on the allowed form of such correlators expressed in terms of the coordinates of G-analytic superspace $x_{A}, \theta^{+}, \bar{\theta}^{+}, u^{ \pm}$. However, such arguments cannot predict the dependence on the gauge coupling constant. The latter can be very efficiently determined by using the reduction formula of ref. [14] (for an explanation in the context of $N=2$ harmonic superspace see [16]). This formula relates the derivative of the 4-point correlator (25) with respect to the (complex) coupling constant $\tau$ to a $4+1$-point correlator obtained by inserting the $N=2$ SYM action:

$$
\frac{\partial}{\partial \tau}\left\langle p_{1} p_{2} p_{3} p_{4}\right\rangle \sim \int d^{4} x_{L 0} d^{4} \theta_{0}\left\langle\operatorname{tr} W^{2}(0)\left(q^{+}\right)^{p_{1}}(1) \ldots\left(q^{+}\right)^{p_{4}}(4)\right\rangle .
$$

Here $W\left(x_{L}, \theta^{i \alpha}\right)$ is the field strength of $N=2 \mathrm{SYM}$ and $L_{N=2} S Y M=-\frac{1}{4} \operatorname{tr} W^{2}$ is the corresponding Lagrangian. Note that unlike the matter superfields $q^{+}$which are G-analytic and harmonic-dependent off shell, $W$ is chiral and harmonic-independent. The integral in the reduction formula (32) goes over the chiral insertion point 0 . As we shall see later on, the combination of chirality with G-analyticity, in addition to conformal supersymmetry and H-analyticity, turns out to be extremely restrictive.

So, from now on we shall concentrate on the $4+1$-point correlators

$$
\left\langle 0 p_{1} p_{2} p_{3} p_{4}\right\rangle
$$

which are chiral at point 0 and $\mathrm{G}$-analytic at points $1, \ldots, 4$, have the corresponding superconformal properties and are also H-analytic,

$$
D_{r}^{++}\left\langle 0 p_{1} p_{2} p_{3} p_{4}\right\rangle=0, \quad r=1, \ldots, 4 \quad \text { if point } 0 \neq \ldots \neq \text { point } 4 .
$$

In addition, they carry a certain $R$-weight. Indeed, the expansion of the matter superfield $q^{+}=\phi^{i}(x) u_{i}^{+}+\ldots$ starts with the physical doublet of scalars of the $N=2$ hypermultiplet which have no $R$-weight. On the contrary, the $N=2 \mathrm{SYM}$ field strength $W=\ldots+\theta \sigma^{\mu \nu} \theta F_{\mu \nu}(x)$ contains the YM field strength $F_{\mu \nu}(R$-weight 0$)$ in a term with two left-handed $\theta$ 's, so the $R$ weight of $W$ equals 2 and that of the Lagrangian equals 4 . ¿From (32) it is clear that this weight is compensated by that of the chiral superspace measure $d^{4} x_{L} d^{4} \theta$, so the correlator in the left-hand side of eq. (32) is weightless.

The task now is to explicitly construct superconformal covariants of $R$-weight 4 out of the coordinates of chiral superspace $x_{L 0}, \theta_{0}^{i \alpha}$ at the insertion point 0 and of G-analytic harmonic superspace $x_{A r}, \theta_{r}^{+\alpha}, \bar{\theta}_{r}^{+\dot{\alpha}}, u_{r i}^{ \pm}, r=1, \ldots, 4$ at the matter points. To this end we need to know the transformation properties of these coordinates under $Q$ and $S$ supersymmetry (parameters $\epsilon$ and $\eta$, correspondingly) 19 :

$$
\begin{aligned}
\delta x_{L}^{\alpha \dot{\alpha}} & =-4 i \theta^{i \alpha} \bar{\epsilon}_{i}^{\dot{\alpha}}-4 i \theta^{i \alpha} x_{L}^{\beta \dot{\alpha}} \eta_{\beta i} \\
\delta \theta^{i \alpha} & =\epsilon^{i \alpha}+x_{L}^{\alpha \dot{\beta}} \bar{\eta}_{\dot{\beta}}^{i}+4 i \theta^{i \alpha} \theta^{j \beta} \eta_{j \beta}
\end{aligned}
$$




$$
\begin{aligned}
\delta x_{A}^{\alpha \dot{\alpha}} & =-4 i u_{i}^{-}\left(\epsilon^{i \alpha} \bar{\theta}^{+\dot{\alpha}}+\theta^{+\alpha} \bar{\epsilon}^{i \dot{\alpha}}\right)+4 i\left(x_{A}^{\alpha \dot{\beta}} \bar{\theta}^{+\dot{\alpha}} \bar{\eta}_{\dot{\beta}}^{i}-x_{A}^{\beta \dot{\alpha}} \theta^{+\alpha} \eta_{\beta}^{i}\right) u_{i}^{-} \\
\delta \theta^{+\alpha} & =u_{i}^{+} \epsilon^{i \alpha}+x_{A}^{\alpha \dot{\beta}} \bar{\eta}_{\dot{\beta}}^{i} u_{i}^{+}-2 i\left(\theta^{+}\right)^{2} \eta^{i \alpha} u_{i}^{-} \\
\delta \bar{\theta}^{+\dot{\alpha}} & =u_{i}^{+} \bar{\epsilon}^{i \dot{\alpha}}-x_{A}^{\beta \dot{\alpha}} \eta_{\beta}^{i} u_{i}^{+}+2 i\left(\bar{\theta}^{+}\right)^{2} \bar{\eta}^{i \dot{\alpha}} u_{i}^{-} \\
\delta u_{i}^{+} & =4 i\left(\theta^{+\alpha} \eta_{\alpha}^{j}+\bar{\eta}_{\dot{\alpha}}^{j} \bar{\theta}^{+\dot{\alpha}}\right) u_{j}^{+} u_{i}^{-} \\
\delta u_{i}^{-} & =0 .
\end{aligned}
$$

We remark that $Q$ supersymmetry acts as a simple shift of the Grassmann variables, whereas $S$ supersymmetry is non-linear. Nevertheless, part of the $S$ transformation is shift-like (the terms $x_{L}^{\alpha \dot{\beta}} \bar{\eta}_{\dot{\beta}}^{i}$ in (35) and $x_{A}^{\alpha \dot{\beta}} \bar{\eta}_{\dot{\beta}}^{i} u_{i}^{+}, x_{A}^{\beta \dot{\alpha}} \eta_{\beta}^{i} u_{i}^{+}$in (36)), as long as we are allowed to invert the $x$ 's. This is possible due to our choice to keep away from any singularities in $x$-space. Thus, we can use the four left-handed parameters $\epsilon^{i \alpha}$ and $x^{\alpha \dot{\beta}} \bar{\eta}_{\dot{\beta}}^{i}$ to shift away the two left-handed spinors $\theta_{0}^{i \alpha}$ at the chiral point (we count the $U(1)$ projections of the $S U(2)$ doublet $i=1,2$ ) and two of the left-handed $\theta_{r}^{+\alpha}$. This means that our correlators effectively depend on two left-handed spinor coordinates. We can make this counting argument more explicit by forming combinations of the $\theta$ 's which are invariant under $Q$ supersymmetry and under the shift-like part of $S$ supersymmetry. $Q$ supersymmetry obviously suggests to use the combinations

$$
\theta_{0 r}^{\alpha}=\theta_{0}^{i \alpha} u_{r i}^{+}-\theta_{r}^{+\alpha}, \quad \delta_{Q} \theta_{0 r}^{\alpha}=0, \quad r=1, \ldots, 4 .
$$

Then we can form the following two cyclic combinations of three $\theta_{0 r}^{\alpha}$ :

$$
\left(\xi_{12 r}\right)_{\dot{\alpha}}=(12) \rho_{r \dot{\alpha}}+(2 r) \rho_{1 \dot{\alpha}}+(r 1) \rho_{2 \dot{\alpha}}, \quad r=3,4
$$

where

$$
\rho_{r \dot{\alpha}}=x_{0 r}^{-2}\left(x_{0 r} \theta_{0 r}\right) \dot{\alpha}
$$

and $x_{0 r} \equiv x_{L 0}-x_{A r}$ are translation-invariant and $(r s) \equiv u_{r}^{+i} u_{s i}^{+}$are $S U(2)$-invariant combinations of the space-time and harmonic coordinates, correspondingly. It is now easy to check that $\xi_{12 r}$ are completely shift-invariant, i.e.,

$$
\delta_{Q+S} \xi_{12 r}=O\left(\theta^{2}\right) .
$$

Here one makes use of the harmonic cyclic identity

$$
(r s) t_{i}+(s t) r_{i}+(t r) s_{i}=0
$$

Let us now inspect the structure of the correlator (33) more closely. As we noted earlier, it has $R$-weight 4 . In superspace the only objects carrying $R$-weight are the odd coordinates, so the $\theta$ expansion of our correlator must start with the product of four left-handed ones. In other words, the correlator should be nilpotent [15, 16]. Moreover, superconformal covariance requires that the shift-like transformations above do not reduce this number, so we must use all the four components of the shift-invariant combinations (38) (notice that they have $R$-weight 1, even though they are right-handed spinors). Thus, we can write down the leading term in the correlator in the following form:

$$
\left\langle 0 p_{1} p_{2} p_{3} p_{4}\right\rangle=\xi_{123}^{2} \xi_{124}^{2} F^{p_{1}-4\left|p_{2}-4\right| p_{3}-2 \mid p_{4}-2}(x, u)+O\left(\theta^{5} \bar{\theta}\right) .
$$

The coefficient function $F$ depends on the space-time and harmonic variables and carries $U(1)$ charges to match those of the correlator and of the nilpotent prefactor. In the present context 
we are not interested in the purely conformal properties of this function, although it is easy to see that it should depend on the conformally invariant cross-ratios of the $x$ 's (times a certain prefactor which gives the nilpotent term in (42) the required dilation weight).

Before going on we remark that the rest of the expansion (42) is completely determined by superconformal covariance. Indeed, in order to keep the required $R$-weight we have to expand in pairs $\theta \bar{\theta}$. The shift-like transformations above do not mix $\bar{\theta}$ with $\theta$. Further, using the same counting argument as before, we conclude that there exists no nilpotent invariant made out of the four $\bar{\theta}^{+}$'s (chirality at point 0 prevents us from employing $\bar{\theta}_{0}$ ). So, all the higher-order terms in (42) are uniquely related to the leading one by superconformal transformations.

Besides superconformal covariance, the second main requirement on the correlator is $\mathrm{H}$-analyticity (34). Since we have only written out the leading term in the $\theta$ expansion (42), there is no need to take into account the nilpotent part of the harmonic derivative (29) (it only contributes to the next level in the expansion). Then H-analyticity is reduced to a condition concerning the pure harmonic dependence. The general solution of the $\mathrm{H}$-analyticity condition on a harmonic function of charge $p$ is

$$
D^{++} f^{p}\left(u^{ \pm}\right)=0 \Rightarrow\left\{\begin{array}{l}
f^{p}=0 \text { if } p<0 \\
f^{p}=u_{i_{1}}^{+} \ldots u_{i_{p}}^{+} f^{i_{1} \ldots i_{p}} \quad \text { if } p \geq 0
\end{array}\right.
$$

In other words, the solution only exists in the case of a non-negative charge and is a polynomial of degree $p$ in the harmonics $u^{+}$. The coefficient $f^{i_{1} \ldots i_{p}}$ forms an irrep of $S U(2)$ of isospin $p / 2$. In our case, if we impose $\mathrm{H}$-analyticity at a given point, the coefficient $f^{i_{1} \ldots i_{p}}$ can only be made out of the harmonics at the remaining points, since no other object carries $S U(2)$ indices (we have locked $\theta_{0}^{i \alpha}$ away in the combinations $\theta_{0 r}$ ). Consequently, an H-analytic harmonic 4-point function has the general form

$$
(12)^{m_{12}}(13)^{m_{13}}(14)^{m_{14}}(23)^{m_{23}}(24)^{m_{24}}(34)^{m_{34}}, \quad m_{r s} \geq 0 .
$$

Now, in our case we have to take account of the nilpotent prefactor in (42). It would be too strong to demand that the function $F$ be $\mathrm{H}$-analytic with respect to all the variables. The point is that the prefactor contains an overall factor $(12)^{2}$ which can improve the behaviour of the function $F$. Indeed, the detailed expansion of the prefactor has the form, after some fierzing,

$$
\begin{aligned}
\frac{\xi_{123}^{2} \xi_{124}^{2}}{(12)^{2}}= & (34)^{2} \rho_{1}^{2} \rho_{2}^{2}+2(23)(31) \rho_{4}^{2}\left(\rho_{1} \rho_{2}\right)+\frac{4}{3}[(23)(14)+(13)(24)]\left(\rho_{1} \rho_{2}\right)\left(\rho_{3} \rho_{4}\right) \\
& + \text { all permutations }
\end{aligned}
$$

where $\rho_{r}$ were defined in (39). Then we can rewrite the expansion (42) as follows:

$$
\left\langle 0 p_{1} p_{2} p_{3} p_{4}\right\rangle=\frac{\xi_{123}^{2} \xi_{124}^{2}}{(12)^{2}} \mathcal{F}^{p_{1}-2\left|p_{2}-2\right| p_{3}-2 \mid p_{4}-2}(x, u)+O\left(\theta^{5} \bar{\theta}\right)
$$

Since the expression (45) has no harmonic zeros, it cannot suppress any singularities in the new coefficient function $\mathcal{F}$. So, we must require that $\mathcal{F}$ be $\mathrm{H}$-analytic on its own,

$$
D_{r}^{++} \mathcal{F}^{p_{1}-2\left|p_{2}-2\right| p_{3}-2 \mid p_{4}-2}=0, \quad r=1, \ldots, 4 .
$$


We have already seen that the general solution of such a condition, if it exists, is of the form (44) where the total charges at each point should match those of $\mathcal{F}$. Thus we obtain the set of equations

$$
p_{r}-2=\sum_{s \neq r} m_{r s}, \quad r=1, \ldots, 4
$$

with $m_{r s}=m_{s r}$.

The reason why eqs. (48) do not always have a solution is that we are looking for $m_{r s}$ which are non-negative integers. This puts constraints on the allowed values of the correlator's charges $p_{1}, \ldots, p_{4}$. An obvious restriction is that the sum of all charges must be even. Further, it is easy to see that charges such that, e.g.,

$$
p_{4}>p_{1}+p_{2}+p_{3}-4
$$

are ruled out. A special case are the "extremal" correlators of references [1, 3] for which, e.g.,

$$
p_{4}=p_{1}+p_{2}+p_{3}
$$

We also see that no solution exists in the "subextremal" case

$$
p_{4}=p_{1}+p_{2}+p_{3}-2
$$

Finally, we recall that the $4+1$-point correlators of the type considered here are uniquely determined by the leading term in their $\theta$ expansion. Therefore the constraints we have found apply to the entire superfield correlation function $\left\langle 0 p_{1} p_{2} p_{3} p_{4}\right\rangle$, and, by means of the reduction formula (32), to the $N=2$ matter correlators $\left\langle p_{1} p_{2} p_{3} p_{4}\right\rangle$. The conclusion is that neither the extremal nor the subextremal four-point correlators receive any quantum corrections beyond tree level. It should be mentioned that the method explained in section 2 leads to a slightly weaker condition on the coefficient functions of the subextremal correlators. It takes the form of a second-order PDE in the space-time variables which requires boundary conditions in order to fix the solution. In this context one might speculate that the reduction formula automatically takes account of some additional field-theory input which is harder to formulate in an approach based on symmetries alone.

To summarise, we have seen that the simple expressions for extremal correlators which were derived in AdS supergravity in [1] can be easily understood on the field theory side as a consequence of analyticity in the harmonic superspace formalism. Furthermore, non-perturbative nonrenormalisation theorems can be proven subject to the assumption that there are no unsuspected contact terms of the type which could interfere with the application of the reduction formula [17]. Strong arguments in favour of this assumption have been given in [16], including an explicit verification of the reduction formula at two loops in the symmetric case $p_{1}=\ldots=p_{4}=2$.

We would also like to emphasise that these results hold for analytic correlators in any $N=2$ SCFT and not just $N=4$, and for any choice of the gauge group. We further believe that these results can be extended to extremal correlators with an arbitrary number of points, although a complete proof would require a more detailed study of nilpotent covariants. This topic is under investigation.

Acknowledgements: CS would like to thank the Institute for Advanced Study, Princeton, for hospitality during the final stage of this project. ES and PW profited from stimulating 
discussions with M. Bianchi and E. D'Hoker. This work was supported in part by the BritishFrench scientific programme Alliance (project 98074), by the EU network on Integrability, nonperturbative effects and symmetry in quantum field theory (FMRX-CT96-0012) and by the grant INTAS-96-0308.

\section{References}

[1] E. D'Hoker, D.Z. Freedman, S. D. Mathur, A. Matusis and L. Rastelli, Extremal correlators in the AdS/CFT correspondence, MIT-CTP-2893, hep-th/9908160.

[2] J. Maldacena, The large $N$ limit of superconformal field theories and supergravity, Adv. Theor. Math. Phys. 2 (1998) 231-252, hep-th/9711200; S.S. Gubser, I.R. Klebanov and A.M. Polyakov, Gauge theory correlators from noncritical String theory, Phys. Lett. B428 (1998) 105, hep-th/9802109; E. Witten, Anti-de Sitter space and holography, Adv. Theor. Math. Phys. 2 (1998) 253-291, hep-th/9802150.

[3] M. Bianchi and S. Kovacs, Nonrenormalization of extremal correlators in $N=4 S Y M$ theory, ROM2F-99-31, hep-th/9910016.

[4] B. Conlong and P. West, Anomalous dimensions of fields in a supersymmetric quantum field theory at a renormalization group fixed point, J. Phys. A26 (1993) 3325.

[5] A.Galperin, E.Ivanov, S.Kalitzin, V.Ogievetsky and E.Sokatchev, Unconstrained $N=2$ matter, Yang-Mills and supergravity theories in harmonic superspace, Class. Quant. Grav. 1 (1984) 469.

[6] G.G. Hartwell and P.S. Howe (N, p, q) harmonic superspace Int. J. Mod. Phys. A10 (1995) 3901-3920, hep-th/9412147; A superspace survey, Class. Quant. Grav. 12 (1995) 1823-1880.

[7] P.S. Howe and P.C. West, Non-perturbative Green's functions in theories with extended superconformal symmetry, hep-th/9509140; OPEs in four-dimensional superconformal field theories, Phys. Lett. B389 (1996) 273-279, hep-th/9607060; Is $N=4$ Yang-Mills soluble? (1996) in Moscow 1996 Physics, 622-626, hep-th/9611074.

[8] P.S. Howe and P.C. West, Superconformal invariants and extended supersymmetry, Phys. Lett. B400 (1997) 307-313, hep-th/9611075.

[9] P.S. Howe, E. Sokatchev and P.C. West, Three-point functions in N=4 Yang-Mills, Phys. Lett. B444 (1998) 341-351, hep-th/9808162.

[10] S. Lee, S.Minwalla, M. Rangamani and N. Seiberg, Three-point functions of chiral operators in $D=4, N=4 S Y M$ at large N, Adv.Theor.Math.Phys 2 (1998) 697-718, hep-th/9806074; E. D'Hoker, D.Z.Freedman and W. Skiba, Field theory tests for correlators in the AdS/CFT correspondence, Phys. Rev. D59 (1999) 45008, hep-th/9807098; F.Gonzalez-Rey, B.Kulik and I.Y.Park, Non-renormalisation of two and three point correlators of N=4 SYM in $N=1$ superspace, Phys. Lett. B455 (1999) 164-170, hep-th/9903094; K. Intriligator and W. Skiba, Bonus symmetry and the operator product expansion of $N=4$ Super-Yang-Mills, hep-th/9905020.

[11] L. Andrianopoli and S. Ferrara, $K-K$ excitations on $A d S_{5} \times S^{5}$ as $N=4$ "primary" superfields, Phys. Lett. B430 (1998) 248-253, hep-th/9803171. 
[12] F.Gonzalez-Rey, I.Park and K.Schalm, A note on four-point functions of conformal operators in $N=4$ super-Yang Mills, Phys. Lett. B448 (1999) 37-40, hep-th/9811155; B. Eden, P.S. Howe, C. Schubert, E. Sokatchev and P.C. West, Four-point functions in N=4 supersymmetric Yang-Mills theory at two loops, Nucl. Phys. B557 (1999) 355-379, hepth/9811172; Simplifications of four-point functions in N=4 supersymmetric Yang-Mills theory at two loops, to appear in Phys. Lett. B, hep-th/9906051.

[13] B. Eden, P.S. Howe, A. Pickering, E. Sokatchev and P.C. West, in preparation.

[14] K. Intriligator, Bonus symmetries of $N=4$ super-Yang-Mills correlation functions via AdS duality, Nucl. Phys. B551 (1999) 575-600, hep-th/9811047.

[15] B. Eden, P.S. Howe and P.C. West, Nilpotent invariants in N=4 SYM, to appear in Phys. Lett. B, hep-th/9905085.

[16] P.S. Howe, C. Schubert, E. Sokatchev and P.C. West, Explicit construction of nilpotent covariants in $N=4 S Y M$, KCL-MTH-99-41, hep-th/9910011.

[17] A. Petkou and K. Skenderis, A non-renormalisation theorem for conformal anomalies, hepth/9906030.

[18] A. Galperin, E. Ivanov, V. Ogievetsky and E. Sokatchev, Harmonic supergraphs: Green functions, Class. Quant. Grav. 2 (1985) 601-616; Harmonic supergraphs: Feynman rules and examples, Class. Quant. Grav. 2 (1985) 617-630.

[19] A.Galperin, E.Ivanov, V.Ogievetsky and E.Sokatchev, Conformal invariance in harmonic superspace, preprint JINR E2-85-363 (1985) published in "Quantum Field theory and Quantum Statistics", vol.2, 233-248, A.Hilger, Bristol (1987). 